\title{
On-call service: the role of chemical pathologists
}

\author{
W D NEITHERCUT, * J R PATERSON, R J SPOONER \\ From the Departments of Pathological Biochemistry, ${ }^{*}$ Western Infirmary, and Gartnavel General Hospital, \\ Glasgow, Scotland
}

SUMMARY The workload of the chemical pathology advisory on-call service was investigated. Over three years 317 calls were recorded, giving a mean of nine calls a month. Seasonal variation in the number of calls was observed, with an increase in calls during December, January, and February. Requests for the arrangement of analysis accounted for 231 calls, while there were 98 requests for advice. Authorisation of a request for analysis was the outcome of 156 calls. Advice with respect to investigation, treatment, or interpretation of results was offered during 173 calls. The acute medical and surgical wards, the accident and emergency department, renal unit and intensive care unit accounted for 198 of the calls. The two analyses most frequently requested, through the advisory oncall service, were serum digoxin and blood ethanol, with 51 and 33 requests, respectively. The "acute" wards and accident and emergency department had the greatest request rate, accounting for 35 of the requests for digoxin and 26 of the requests for blood ethanol.

The acute care areas were responsible for the major part of the workload, and a clinical requirement for the services of chemical pathologists out of hours, was observed.

Clinical chemistry laboratories usually operate both an analytical on-call service provided by medical laboratory scientific officers (MLSOs) and a senior staff or advisory on-call rota. ${ }^{1}$ In our experience the role of the senior staff on-call rota may vary from laboratory to laboratory. In some laboratories the senior staff or advisory rota is responsible for vetting all requests for out of hours clinical chemistry, while in others its function is to protect the on-call MLSO from unreasonable or excessive demands for clinical chemistry. The senior staff rota is also often used as the mechanism whereby requests for clinical chemistry tests outside the range of tests available directly from the on-call MLSO may be instigated. The senior staff rota may also provide specialist investigations and advice with regard to the interpretation of results, and investigation and management of patients.

Laboratory medical staff and scientists may be responsible for providing the senior staff on-call service. In district general hospital laboratories consultant chemical pathologists or top grade scientists may provide this. In teaching hospitals it may be the duty of junior medical staff and other grades of scientists in training. Many laboratories have an informal system of referral of requests to senior staff on-call.' $A$ list of telephone numbers may be available with the instruction that each number should be tried

Accepted for publication 11 July 1989 in turn until one of the senior staff is contacted. In some laboratories the advisory on-call service may be organised with strict adherence to a rota.

The analytical on-call service provided by MLSOs has been investigated by several groups. ${ }^{1-9}$ The cost effectiveness of the analytical service and alternative arrangements for the analysis of clinical chemistry samples out of hours have been the main interest of investigators. The workload and pattern of clinical use of senior staff on-call services, however, has not been investigated. We therefore thought it useful to investigate the source, incidence, nature and outcome of requests received by the advisory on-call rota in our hospital to see if a chemical pathologist was needed out of hours.

\section{Methods}

In our laboratory, the advisory on-call service operated in parallel with the analytical service staffed by MLSOs. A limited list of tests was available directly by arrangement with the on-call MLSO (table 1). Any request beyond the scope of this list was referred to the advisory on-call staff. The personnel responsible for providing the advisory on-call service were expected to adhere to the rota and be available on standby during the dates assigned to them. During the period of the study the grades of staff responsible for the provision of the advisory service were senior registrar and 
Table 1 Analyses available directly from on-call MLSO

\begin{tabular}{ll}
\hline Serum & Sodium \\
& Potassium \\
Bicarbonate & Urea \\
& Creatinine \\
& Amylase \\
Salicylate \\
Paracetamol \\
Phenobarbitone \\
& Calcium \\
& Iron \\
& Glucose \\
& Arterial blood gases \\
& Packed cell volume \\
Heparinised blood & Urine osmolality \\
& Cerebrospinal fluid glucose \\
\hline
\end{tabular}

registrar in chemical pathology and principal and senior biochemists.

The study was conducted over three years from March 1986 to February 1989. During the study all calls received by the advisory service were logged. The date and time of calls were recorded as were the ward from which the call originated, purpose of the call, advice offered by the staff of the advisory service, and any analyses authorised.

The on-call service in our department is termed an advisory rota as a consultant chemical pathologist or equivalent grade scientist did not participate in the rota. The terms advisory on-call service and senior staff on-call service are, however, used interchangeably in this paper.

\section{Results}

Three hundred and seventeen calls to the advisory oncall service were recorded. In the first year there were 94 calls, in the second 118 , and in the third 105 , giving a mean of nine calls each month. A seasonal variation in the number of calls each month was observed. More calls were made in December, January, and February (table 2).

One hundred and ninety eight $(61 \%)$ calls came from the acute care areas of the hospital. These included the acute medical wards, the accident and emergency department, the intensive care unit, the renal unit and the acute surgical wards. The acute medical wards generated the greatest number of calls with $61(21 \%)$, followed by the accident and emergency department $57(18 \%)$, the intensive care unit 21 $(6 \%)$, the renal unit $18(5 \%)$, and the acute surgical wards with $12(4 \%)$ calls.

The commonest reason for contacting the advisory on-call staff was to arrange for analysis of a sample (table 3). This occurred during 231 (73\%) of the calls. A request for advice was the reason for contacting the advisory service on $98(31 \%)$ occasions. While arrangement of analysis was the major reason for a call, the analysis requested was authorised on 156 $(68 \%)$ occasions. Conversely, while advice was requested during $98(31 \%)$ calls the staff providing the advisory service considered it appropriate to offer advice during $173(55 \%)$ calls.

The acute care areas were responsible for $23(31 \%)$ of the requests for advice. The other calls, which initially requested advice, were distributed throughout the other units of the hospital. The maximum number of requests for advice from any single unit was 13 $(18 \%)$ calls from the acute medical wards. During the three years of the study the number of calls requesting advice rose from $23(24 \%)$ in the first 12 months to 37 $(35 \%)$ in the last 12 months. The advice offered during a call was categorised. Advice was given with respect to the interpretation of results, investigation of patients, and treatment. Both the proportion and absolute number of calls offered advice concerning the treatment of patients increased from $13(14 \%)$ in the first 12 months to $24(23 \%)$ in the final 12 months. Most of the advice offered to clinicians with respect to treatment (33 calls or $60 \%$ ) concerned the management of fluid and electrolyte disturbances.

Table 4 lists the tests commonly requested of the advisory service. The total number of tests requested was greater than the number of calls initially requesting an analysis. This was due to multiple requests for analysis during some calls. For the total of 250 analyses listed, $173(69 \%)$ analyses were arranged. Serum digoxin and blood alcohol concentrations were most frequently requested. The acute care areas made $35(69 \%)$ of the requests for digoxin analysis, while the accident and emergency department accounted for 26 $(79 \%)$ of the requests for blood ethanol. The proportion of requests denied by the advisory service varied from analyte to analyte. Requests for creatine kinase activity and drug screens were commonly turned down. Urine osmolality and serum lactate measurement were authorised more frequently than

Table 2 Mean number of cells received by advisory on-call service each month

\begin{tabular}{llllllllllllll}
\hline & Jan & Feb & Mar & April & May & June & July & Aug & Sept & Oct & Nov & Dec \\
\hline No of calls & 15 & 13 & 7 & 10 & 9 & 7 & 8 & 7 & 7 & 8 & 6 & 12 \\
\hline
\end{tabular}


Table 3 Analysis of requests made to on-call service

\begin{tabular}{lll}
\hline & No of & $\begin{array}{l}\text { Outcome of } \\
\text { request: analyses } \\
\text { authorised, advice } \\
\text { offered }\end{array}$ \\
\hline requests & 231 & 156 \\
\hline $\begin{array}{l}\text { Analyses requested by clinician } \\
\text { Analyses initiated by advisory } \\
\text { staff }\end{array}$ & 41 \\
$\begin{array}{l}\text { Advice } \\
\text { Complaint }\end{array}$ & 98 & 173 \\
\hline
\end{tabular}

Table 4 Tests frequently requested by clinicians and tests authorised by advisory on-call service

\begin{tabular}{lcc}
\hline & No of & $\begin{array}{l}\text { No (\%) of } \\
\text { requests } \\
\text { authorised }\end{array}$ \\
\hline requests & 51 & $29(56)$ \\
Digoxin & 33 & $17(52)$ \\
Alcohol & 24 & $23(96)$ \\
Cerebrospinal fluid protein & 21 & $17(81)$ \\
Theophylline & 21 & $13(62)$ \\
Phenytoin & 16 & $16(100)$ \\
Methotrexate & 15 & $20(134)$ \\
Urine osmolality & 10 & $8(80)$ \\
Carboxyhaemoglobin & 10 & $9(90)$ \\
Fluid electrolytes & 10 & $7(70)$ \\
Carbamazepine & 10 & $1(10)$ \\
CK/CKMB & 9 & $2(22)$ \\
TLC drug screen & 5 & $5(100)$ \\
Amylase creatinine clearance ratio & 4 & 0 \\
Barbiturates & 4 & $1(25)$ \\
Lithium & 4 & $1(25)$ \\
Paraquat & 3 & $4(133)$ \\
Lactate & & $173(69)$ \\
& 250 & \\
\hline
\end{tabular}

requested by the clinicians. Some of these tests were authorised following an initial request for advice, while others were authorised following the substitution of an inappropriate investigation with a more appropriate investigation after discussion of the reasons for the request.

The on-call MLSOs contacted the advisory service on 26 occasions during the study. Twenty one $(81 \%)$ of these calls were to pass on results that the MLSO considered worthy of follow up, either because they were noticeably abnormal or because the MLSO had gained the impression that the clinician who had requested the tests would benefit from advice with respect to their interpretation, or advice concerning further investigation of the patient. As a result of these calls from MLSOs, 16 hyponatraemic patients, all with serum sodium concentrations of less than 113 $\mathrm{mmol} / \mathrm{l}$, and two hypokalaemic patients were followed up by the advisory on-call staff. On five occasions, however, the on-call MLSO contacted the advisory service because of an apparent abuse of the analytical service by clinical staff.

\section{Discussion}

This study provides evidence for the need for an out of hours chemical pathology advisory service. This conclusion is supported by the observations that the acute care areas were responsible for $61 \%$ of the requests for the arrangement of analysis and were also the areas which most frequently requested the advice of a chemical pathologist. The availability of experienced clinical staff in the acute care areas may have been the reason that a proportionately greater number of analyses were requested than advice sought.

Before the study it had been thought that the pattern of calls received would reflect the turnover of junior medical staff in the hospital, with an increase in calls occurring in February and August while newly appointed junior house officers were discovering how to use the chemical pathology services. Variation in the use of the advisory on-call service was observed. The highest rates occurred during the months of December, January, and February. The admission workload of acute care areas increases during the winter months and this increase in use of the advisory service probably reflected an increase in the acute medical and surgical workload of the hospital.

Legg reported that $80 \%$ of requests for analysis referred to senior laboratory staff were authorised.' We found that $49 \%$ of all calls resulted in the analysis requested being authorised, or that $68 \%$ of requests for analysis resulted in authorisation of the test requested. Tests were also arranged following a request for advice. This resulted in the authorisation of 41 additional tests. A maximum of 197 calls was therefore followed by an analysis, representing $63 \%$ of all calls. The total number of tests authorised might have been lower as some tests, including blood ethanol and creatine kinase activity, were authorised on some occasions not for their clinical necessity but for the information and education of the requesting clinician. Some tests were rarely authorised. This included requests for serum creatine kinase activity or creatine kinase isoenzmes, urine drug screens, and requests for total barbiturates or specific barbiturates other than phenobarbitone. Requests for these tests may indicate a failure to appreciate the role of these tests in the immediate management of acutely ill patients. Other tests including serum methotrexate, serum theophylline, and cerebrospinal fluid protein concentrations were almost always authorised, indicating that these could be added to the limited list of tests directly available from the MLSO.

The advisory on-call staff therefore played an important part in determining the outcome of a 
request for an analysis.

Specific requests for advice accounted for $31 \%$ of the total number of calls during the study. In $84 \%$ of these calls advice regarding the interpretation of results was sought and in the remaining $16 \%$ treatment of patients was discussed. The latter mainly concerned the management of fluid and electrolyte disorders such as hyponatraemia and hyperkalaemia.

Advice was offered during $55 \%$ of all calls and in the last 12 months of the study $23 \%$ of calls offered advice concerning the management of patients, indicating the importance of having medically qualified chemical pathologists on-call.

A senior staff or advisory on-call service is often perceived as a method of protecting the on-call MLSO from excessive or unnecessary work. This did not seem to be a major problem as only five calls were requests from the MLSO for help in dealing with junior doctors who were considered to be abusing the analytical oncall system.

In conclusion, we found that there was a genuine clinical need for chemical pathologists out of hours. Their role included interpretation of results and participation in patient management as well as the arrangement of analyses.
References

1 Legg EF. The on-call service: a regional survey. Ann Clin Biochem 1989;26:19-25.

2 Smith AD, Shenkin RA, Dryburgh FJ, Morgan HG. Emergency biochemistry services-are they abused? Ann Clin Biochem 1982;19:325-8.

3 Morgan DB, Gunn IR, Faye S, Clegg G, Grant AM. A comparison of alternative arrangements for an out-of-hours chemical pathology service. Lancet 1985;i:859-61.

4 Lester E. Out-of-hours laboratory investigations. Lancet 1985;ii:500.

5 Young RM, Payne RB. Effectiveness of out of hours laboratory investigation. Br Med J 1981;283:289-91.

6 Lester E. A new strategy for out-of-hours laboratory investigations. Ann Clin Biochem 1986;23:497-500.

7 Smith JM, Guest MT. "On-call"- the Croydon solution. Ann Clin Biochem 1987;24(suppl 1):56-7.

8 Fraser CG, Woodford FP. Strategies to modify the test-requesting patterns of clinicians. Ann Clin Biochem 1987;24:223-31.

9 Gunn IR, Little AJ, Payne R. Effects of workload and analysis time as the cost of out-of-hours investigations. Ann Clin Biochem 1986;23:501-3.

Requests for reprints to: Dr W D Neithercut, Department of Pathological Biochemistry Western Infirmary, Glasgow G1 1 6NT, Scotland. 